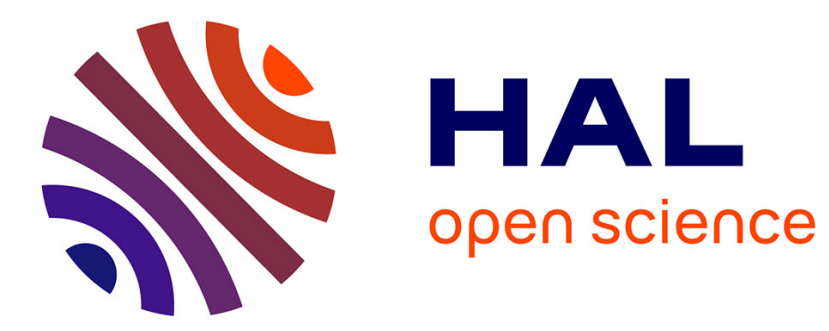

\title{
Bruit des avions légers : réduction du rayonnement acoustique d'une hélice
}

\author{
M. Chusseau, A. Carrère, P. Matharan, S. Pauzin
}

\section{To cite this version:}

M. Chusseau, A. Carrère, P. Matharan, S. Pauzin. Bruit des avions légers : réduction du rayonnement acoustique d'une hélice. Journal de Physique IV Proceedings, 1994, 04 (C5), pp.C5-993-C5-996. 10.1051/jp4:19945218. jpa-00252903

\section{HAL Id: jpa-00252903 https://hal.science/jpa-00252903}

Submitted on 1 Jan 1994

HAL is a multi-disciplinary open access archive for the deposit and dissemination of scientific research documents, whether they are published or not. The documents may come from teaching and research institutions in France or abroad, or from public or private research centers.
L'archive ouverte pluridisciplinaire HAL, est destinée au dépôt et à la diffusion de documents scientifiques de niveau recherche, publiés ou non, émanant des établissements d'enseignement et de recherche français ou étrangers, des laboratoires publics ou privés. 


\title{
Bruit des avions légers : réduction du rayonnement acoustique d'une hélice
}

\author{
M. CHUSSEAU, A. CARRÈRE* ${ }^{*}$ P. MATHARAN et S. PAUZIN*
}

SOCATA, BP. 930, 65009 Tarbes, France

* CERT/DERMES, BP. 4025, 31055 Toulouse, France

\begin{abstract}
résumé : This paper deals with an acoustic and aerodynamic parametrical study of a light airplane propeller. The aim of this study is to obtain a decrease of noise level with checking the performances. Flyover sound generation is analysed through an analytical formulation which is a sum of blade passing frequency harmonics. D. B. Hanson's formulation used for sound pressure level calculations is coupled with aerodynamic investigations which are carried out with a model based on lifting line theory. In the first part, the accuracy of the acoustic method is achieved with experimental measurement comparison. Next, propeller geometry parametrical study shows the influence of number of blades and scale blade factor modifications. The most suitable solution to provide the same performances with a reduction of noise is obtained by performing the best compromise between acoustics and aerodynamics.
\end{abstract}

\section{INTRODUCTION}

En Europe, les nouveaux règlements de certification acoustique des avions monomoteurs à pistons deviennent de plus en plus sévères. Par souci de protection de l'environnement, la réduction des nuisances sonores extérieures de l'avion sont donc nécessaires. Le bruit émis par ce type d'avion à hélice est un bruit de raies dont la fréquence du fondamental est proportionnelle à la fréquence de rotation de l'arbre hélice [1]. L'énergie contenue dans ces raies provient de l'hélice et de l'échappement qui sont les deux principales sources sonores. Une étude expérimentale composée d'essais au sol et en survol a montré que l'hélice est la source de bruit prépondérante [2]. L'objectif de l'étude paramétrique aérodynamique et acoustique de l'hélice présentée dans cet article est d'obtenir une réduction du rayonnement acoustique de l'hélice sans dégradation des performances aérodynamiques.

Partant du principe qu'une source acoustique est le résultat d'un mécanisme oscillatoire où l'air est soumis à de petites variations de masse et de quantité de mouvement, on montre que le rayonnement acoustique d'une hélice est induit par le mouvement des pales et par les interactions aérodynamiques qui en résultent. Le comportement aérodynamique de l'hélice est une des causes de son rayonnement. Ainsi, la détermination du niveau de pression acoustique complétée par le calcul des performances aérodynamiques de la pale comporte deux intérêts : le premier est d'être capable de connaître le niveau sonore pour une configuration aérodynamique spécifique; le second est de suivre l'évolution commune du niveau de bruit et des performances aérodynamiques lors de modifications géométriques de l'hélice.

\section{MODELES ANALYTIQUES}

\subsection{Approche acoustique : formulation de D.B. Hanson}

Afin de pouvoir s'affranchir de la présence des écoulements qui empêchent la résolution des équations classiques de propagation, l'analogie acoustique énoncée par Lighthill consiste à remplacer les surfaces en mouvement par une distribution de sources équivalentes de sorte que les fluctuations réelles de 
pression acoustique soient identiques à celles résultant du rayonnement de ces distributions dans un milieu homogène et au repos [3]. Ces sources sont généralement surfaciques dans le cas du champ acoustique d'une hélice. Elles expriment, d'une part, le mouvement des surfaces sous forme de monopole et, d'autre part, l'interaction des surfaces avec les écoulements sous forme de dipole. Les résolutions analytiques concernant les pales en rotation sont nombreuses [4] et peuvent être effectuées soit dans le domaine temporel soit dans le domaine fréquentiel.

La formulation de D.B. Hanson [5], développée dans un repère fixe (Figure 1 ) donne la pression acoustique à un point observateur sous forme d'harmoniques de la fréquence de passage des pales.

$$
p(t)=\sum_{m=-\infty}^{+\infty} P_{m B} e^{-\frac{i m B \Omega t}{1-M_{x} \cos \theta}} \text { (1) }
$$

L'expression du dipole ou bruit de charges est donnée à travers la distribution en envergure des coefficients de portance $c_{Z}$ et de traînée $c_{X}$.

L'expression du monopole ou bruit d'épaisseur est décrite par la répartition en envergure des profils. Le point d'action des charges se situe à $50 \%$ du bord d'attaque et l'axe de changement de pas est supposé traverser chaque section au milieu de

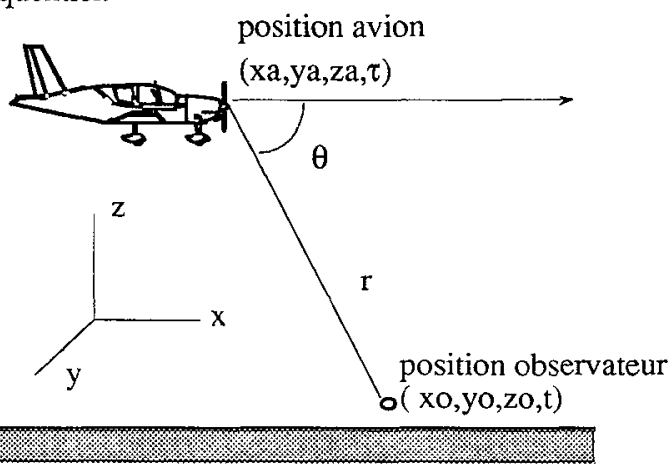

Figure 1: position de l'avion et de l'observateur l'épaisseur du profil.

Ainsi les harmoniques de charges $P c m$ et d'épaisseur $P e m$ dépendent du nombre de pales $B$, du diamètre $D$, de la vitesse d'avancement de l'avion $V_{x}$, de la vitesse de rotation des pales $\Omega r_{O}$ et enfin de la forme en plan et des performances aérodynamiques du profil.

$$
\left\{\begin{array}{l}
P e m \\
P c m
\end{array}\right\}=-\frac{\rho_{0} c_{0}^{2} B D e^{i m B\left(\frac{\Omega_{D} r}{c_{0}}-\frac{\pi}{2}\right.}}{4 \pi r\left(1-M_{x} \cos \theta\right)} \int_{0}^{1} M_{h}^{2}(z) e^{i\left(\phi_{0}+\phi_{j}\right)} J_{m B}\left(\frac{m B z M_{h}(z) \sin \theta}{1-M_{x} \cos \theta}\right)\left\{\begin{array}{l}
k_{x}^{2} e r(z) \Psi_{e}\left(k_{x}\right) d z \\
-i\left(k_{x} \frac{c_{x}}{2} \Psi_{c x}\left(k_{x}\right)+k_{y} \frac{c_{z}}{2} \Psi_{c z}\left(k_{x}\right)\right) d z
\end{array}\right.
$$

où : $\quad-k_{x}$ et $k_{y}$ sont des nombres d'ondes associés à la corde et à l'envergure,

- $\Psi_{e}, \Psi_{c_{x}}$ et $\Psi_{c_{z}}$ sont les transformées de Fourier spatiales des termes sources,

- $\phi_{\mathrm{s}}$ et $\phi_{O}$ sont l'expression des phases créées par une mise en flèche ou par une mise en conicité.

Le niveau de pression acoustique peut être ainsi déterminé pour plusieurs positions lors d'un passage de l'avion au dessus d'un point observateur. L'évolution des sources en fonction de la position relative avion-observateur montre que le niveau maximal apparaît lorsque l'avion est en aval du microphone et que le bruit de charges est la source prépondérante [6]. Le bruit d'épaisseur n'est pas négligeable lorsque l'avion se situe en amont. Ces évolutions sont identiques à celles trouvées dans la littérature[1].

\subsection{Modélisation aérodynamique}

Le progamme de calcul de détermination des performances locales et globales de l'hélice est basée sur la théorie de la ligne portante de Prandtl [7]. Le calcul repose sur un processus itératif portant sur la détermination de la circulation. A partir d'un pas initial de chaque tourbillon libre, la loi de Biot \& Savart permet de connaître la géométrie de la nappe tourbillonnaire, la circulation et les vitesses induites qui lui sont associées :

$$
\vec{V}=-\frac{\mathfrak{l}}{4 \pi} \int_{l \in \varphi} \frac{\boldsymbol{I M} \cdot d \vec{l}}{|\vec{M}|^{3}}
$$

L'angle d'incidence et la vitesse relative de chaque profil ainsi obtenus conduisent à la détermination du fonctionnement local du profil et à la ciculation donnée par le théorème de Kutta-Joukowski :

$$
\Gamma\left(r_{o}\right)=1 / 2 b\left(r_{o}\right) w\left(r_{o}\right) c_{z}\left(\alpha_{i}, M_{h}\right)
$$

La géométrie de la nappe est recalée de manière à assurer la convergence des deux méthodes de calcul de la circulation. Ensuite par intégration sur l'envergure, les coefficients de puissance et de traction de même que l'efficacité de l'hélice sont déterminés. 


\section{ETUDE PARAMETRIQUE}

L'avion étudié est équipé d'un moteur à 4 cylindres avec un régime maximal de $2700 \mathrm{tr} / \mathrm{mn}$ pour une puissance sur arbre de $147 \mathrm{~kW}$. L'hélice bipale à bout carré a un diamètre de $1,88 \mathrm{~m}$. L'ensemble des résultats est présenté en Unités arbitraires pour les niveaux de pression acoustique et en différence de traction pour l'aérodynamique. La configuration de calcul choisie ici représente la procédure de certification : l'avion est en palier à $300 \mathrm{~m}$ sol, le point observateur est à $1,2 \mathrm{~m}$ du sol.

\subsection{Validation expérimentale}

Une comparaison des résultats analytiques obtenus est effectuée avec un spectre de pression acoustique mesuré lors d'un passage en palier de l'avion. La Figure 2 montre les niveaux globaux de pression acoustique (charges + épaisseur) estimés et mesurés, du fondamental et des 5 premiers harmoniques. Pour les trois premiers harmoniques les niveaux mesurés et calculés sont proches. La réflexion du sol induit une modification spectrale du signal. Les fréquences pour lesquelles le signal direct est en opposition de phase avec le signal réfléchi dépendent de la position de l'avion et conduisent à une baisse importante du niveau de bruit. Ces fréquences appelées fréquences d'extinction peuvent être mises en évidence ( harm3, Figure 2) grâce à la prise en compte de la réflexion du sol.

La différence des niveaux mesurés des harmoniques d'ordre élevé peut provenir soit

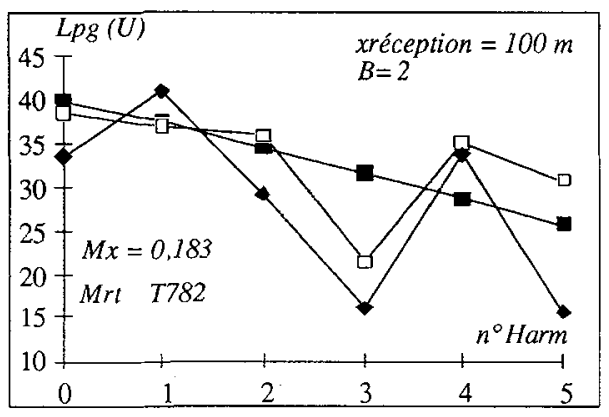

Figure 2: comparaison des niveaux globaux de pression acoustique des harmoniques estimés et

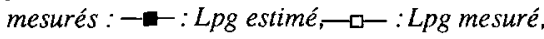
$\longrightarrow$ - Lpg estimé avec réflexion du sol. Niveaux globaw :- $-=42 U,-\square-=43 U$, $\longrightarrow=41 \mathrm{U}$. d'une interaction avec le fuselage, notamment le capot moteur, soit de l'inclinaison de l'hélice par rapport au plan de rotation due au calage moteur.

\subsection{Influence du nombre de pales et du facteur d'échelle de la pale}

Une première étude montre qu'une augmentation du nombre de pales conduit à une réduction sonore et à une chute importante de la traction de l'hélice. Ainsi, afin d'obtenir une diminution du bruit avec maintien des performances, la géométrie de la pale est changée. Le diamètre, la corde et l'épaisseur sont modifiés grâce à un facteur d'échelle de sorte que l'épaisseur relative reste constante. Un nouveau vrillage de la pale est donné dans le but d'obtenir les mêmes angles d'incidence pour chaque section.

La Figure 3 montre les différences de traction obtenues par rapport à l'hélice de base. Pour chaque nombre de pales, on obtient un facteur d'échelle optimal.

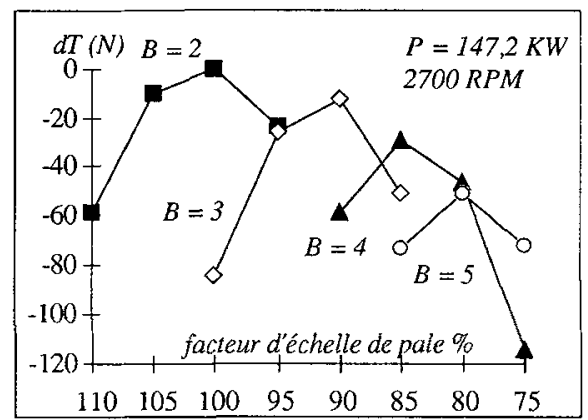

Figure 3: variation de la traction dT en fonction de $B$ et de la grandeur de pale

D'une part, si la surface de la pale devient trop petite, les charges de celle-ci seront trop importantes de sorte que le rendement de l'hélice chutera. D'autre part, une surface d'hélice trop grande conduit à une force de traînée excessive.

La Figure 4 présente la différence entre le niveau global maximal de pression acoustique sur un passage de l'avion et le niveau maximal de l'hélice de base. Une augmentation du nombre de pales aussi bien qu'une diminution de la grandeur conduisent à une réduction du niveau sonore. Une répartition inférieure des charges surfaciques de même qu'une diminution de la vitesse en bout de pale favorisent ce changement. 


\subsection{Analyse et comparaison}

L'analyse des résultats montre que l'évolution acoustique diffère de celle de l'aérodynamique. Pour un nombre de pales donné, l'allègement des charges de la pale qui conduit à une diminution du bruit de charges, ne fournit pas systématiquement une réduction du niveau global. En effet, si la surface de l'hélice est suffisamment grande, le bruit d'épaisseur augmente et devient la source majeure de bruit. Par contre, il est important de noter que chaque optimum se comporte de la même façon : même évolution en envergure de la circulation et du coefficient de portance, même importance relative des sources.

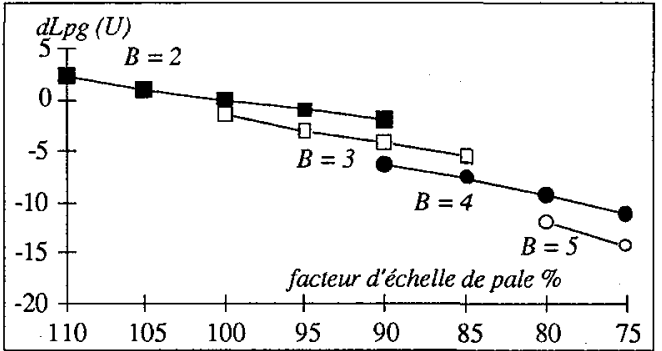

Figure 4: différence de niveau global de pression acoustique en fonction de $B$ et de la grandeur de pale

Un optimum aérodynamique et acoustique est alors obtenu : une hélice tripale avec un facteur d'échelle de la pale de $90 \%$ conduit à une réduction du niveau global de 4 unités pour une diminution de la traction de l'ordre de $0,7 \%$. D'autres paramètres tels que la corde, la mise en flèche sont encore à l'étude et permettront d'effectuer d'autres combinaisons pour une réduction du rayonnement avec un maintien des performances.

\section{CONCLUSION}

Une étude analytique acoustique et aérodynamique d'une hélice d'avion monomoteur à pistons est réalisée. Le calcul des performances aérodynamiques basé sur une méthode de ligne portante fournit les données nécessaires pour l'estimation des niveaux de pression acoustique sous forme d'harmoniques de la fréquence de passage des pales. Pour des modifications de la grandeur et du nombre de pales de l'hélice, l'évaluation corrélative de la traction de l'hélice et du niveau de pression acoustique montre qu'en fonction de la contribution de chaque source sonore (bruit d'épaisseur et bruit de charges), les variations de niveaux de pression ne suivent pas l'évolution des performances. Des solutions conduisant à une réduction sonore avec maintien de performances peuvent être dégagées. L'essai de prototypes actuellement en cours permettra de confirmer ces résultats. D'autre part, de nouvelles hélices conçues à partir de profils différents seront analysées pour fournir de nouvelles optimisations.

\section{Nomenclature:}

$\mathrm{B}:$ nombre de pales

$\mathrm{b}\left(\mathrm{r}_{\mathrm{o}}\right)$ : corde

$c_{O}:$ célérité du son

$c_{\mathrm{X}}$ : coefficient de traînée

$\mathrm{c}_{\mathrm{z}}$ : coefficient de portance

er : épaisseur relative

$m$ : nombre d'harmoniques

$\mathrm{M}_{\mathrm{X}}$ : nombre de Mach

d'avancement

\author{
$\mathrm{M}_{\mathrm{Tl}}$ : nombre de Mach \\ de rotation en bout de pale \\ $\mathrm{M}_{\mathrm{h}}\left(\mathrm{r}_{\mathrm{O}}\right)$ : nombre de Mach relatif \\ $\mathrm{p}$ : pression acoustique \\ $r$ : distance source-observateur \\ $r_{0}$ : position en envergure \\ $\mathrm{V}$ : vitesse induite globale \\ $\mathrm{W}$ : vitesse relative globale \\ $\mathrm{z}$ : position relative en envergure \\ $\alpha$ : angle d'incidence
}

$\Gamma\left(\mathrm{r}_{0}\right)$ : circulation à $\mathrm{r}_{\mathrm{O}}$

$\gamma_{v}$ : intensité du tourbillon

$\rho_{0}$ : masse volumique de l'air

$\theta$ : angle source-observateur

$\Omega$ : vitesse de rotation

$\Omega_{\mathrm{D}}: \Omega / 1-\mathrm{M}_{\mathrm{X}} \cos \theta$

\section{REFERENCES}

[1] H. Gounet, "Contribution à l'étude du bruit des avions légers", Rapport ONERA, 1982.

[2] M. Chusseau, D. Biron, S. Pauzin and P. Matharan, "Light propeller aircraft noise study : experimental approach to identify the contribution of the main sources", Acoustics'93, Southampthon,UK, avril 1993, Proc.I.O.A. Part 3, pp. 225-232.

[3] M.E. Goldstein, Aeroacoustics, (Mc Graw-Hill International Book Co. 1976).

[4] F. Farassat, "Linear acoustic formulas for calculation of rotating blade noise", AIAA Journal, vol. $19, \mathrm{n}^{\circ} 9$ (1981), pp. 1122-1130.

[5] D.B. Hanson, "The influence of propeller design parameters on far field harmonic noise in forward flight", 5th Aeroacoustics Conference, US, 1979, AIAA paper 79-0609.

[6] M. Chusseau, E. Roozen, S. Pauzin, P. Matharan and A. Carrère, "Light aircraft propeller noise study : design parameter effects on acoustics and aerodynamics", AIAApaper 93-4443.

[7] M. Courjaret, M. Desplanches, "Aerodynamique des rotors de convertibles", Paris 1974, Aerospatiale. 